-Supporting Information-

\title{
Measuring Cellular Uptake of Polymer Dots for Quantitative Imaging and Photodynamic Therapy
}

Ye Yuan, ${ }^{1}$ Weiying Hou, ${ }^{2}$ Zezhou Sun, ${ }^{2}$ Jie Liu, ${ }^{2}$ Ning Ma, ${ }^{2}$ Xiaosong Li, ${ }^{3}$ Shengyan Yin, ${ }^{1}$ Weiping Qin, ${ }^{1}$ and Changfeng $\mathrm{Wu}^{*, 2}$

'State Key Laboratory on Integrated Optoelectronics, College of Electronic Science and Engineering, Jilin University, Changchun, Jilin 130012, China ${ }^{2}$ Department of Biomedical Engineering, Southern University of Science and Technology, Shenzhen, Guangdong 518055, China 3Department of Oncology, Fourth Medical Center of Chinese People's Liberation Army General Hospital, Beijing 100048, China

* To whom correspondence should be addressed E-mail:wucf@sustech.edu.cn

Phone: 0755-88015150 


\section{Contents}

\section{Supplementary Figures}

Figure S1 Spectroscopic characterization of photosensitizer PtOEP and its spectral overlap with polymer PFBT.

Figure S2 Fluorescence stability of PFBT Pdots and PFBT@Pt Pdots.

Figure $S_{3}$ Brightness comparisons of MCF-7 cells labeled with Pdots by flow cytometry and cytotoxicity assay of Pdots.

Figure S4 Typical confocal microscopy images with integrated intensity over Z-stacking images of PFBT-labeled MCF-7 cells.

Figure $\mathbf{S}_{\mathbf{5}}$ Changes of absorption spectra of ADMA in the presence of Rose Bengal, PFBT@Pt Pdots, and ADMA by light irradiation.

Figure S6 Confocal fluorescence imaging of intracellular ${ }^{1} \mathrm{O}_{2}$ detection 
a)

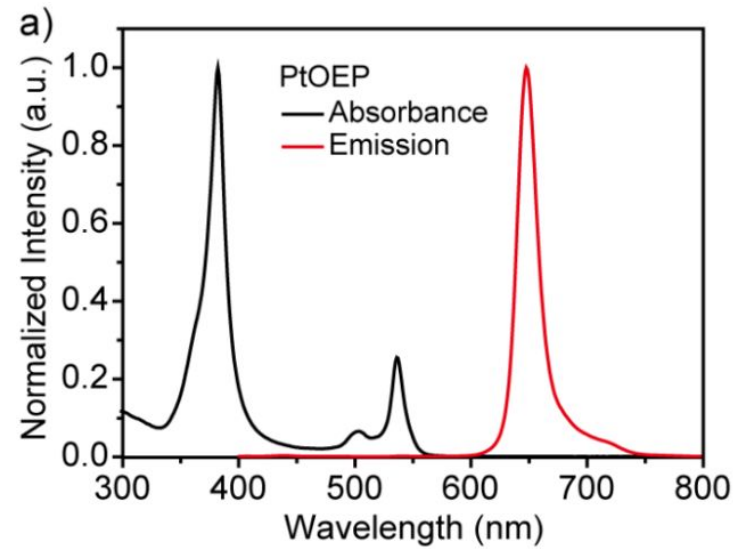

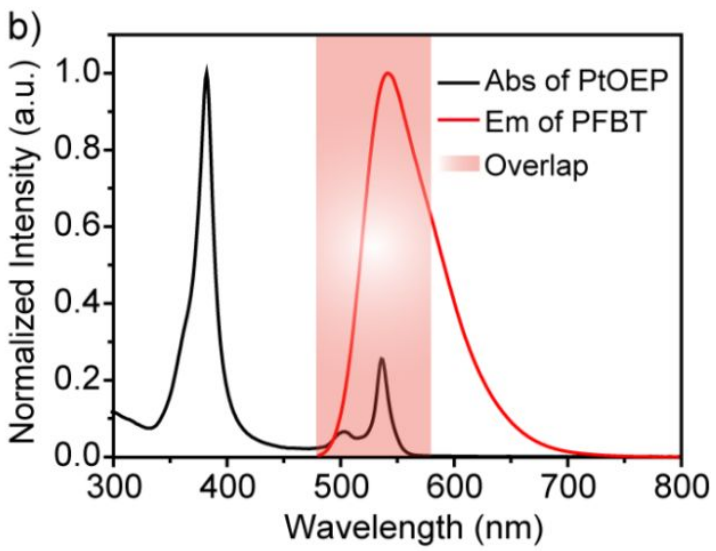

Figure S1. a) Absorption and emission spectra of photosensitizer dye PtOEP. b) Spectral overlap between the emission of polymer PFBT and the absorption of dye PtOEP.

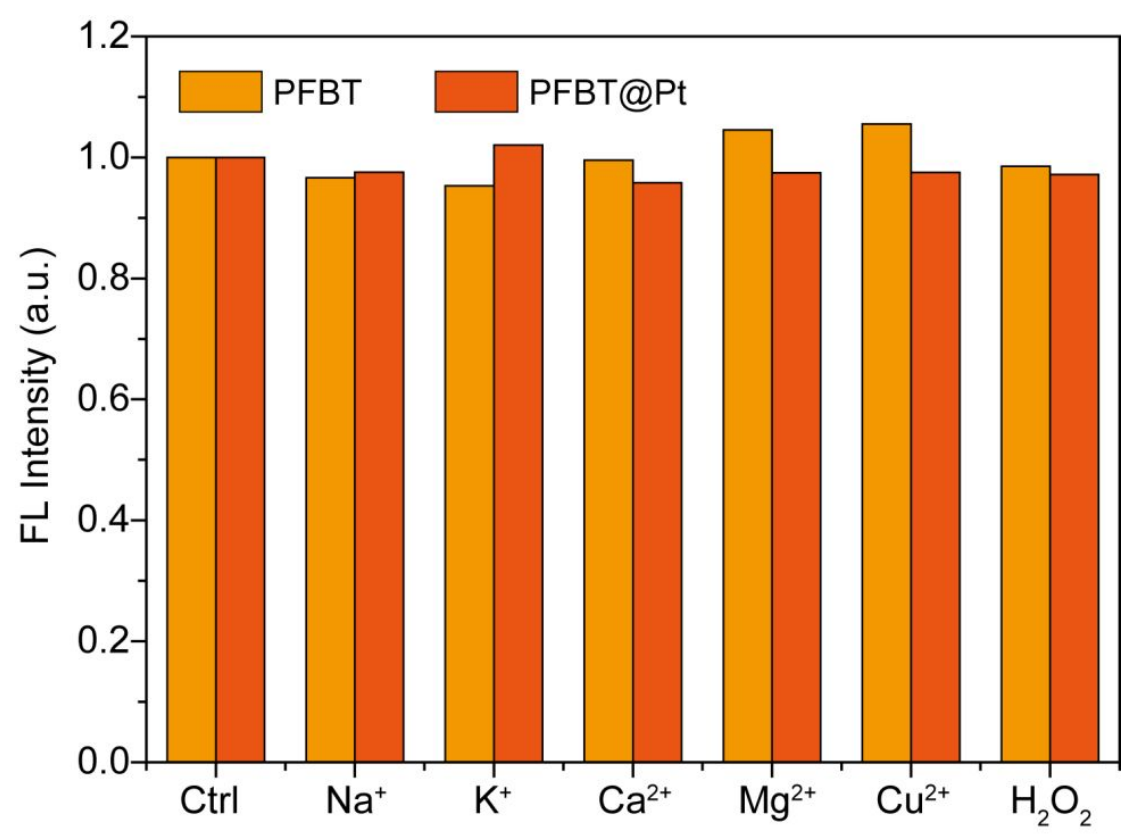

Figure S2. The fluorescence stability of PFBT Pdots and PFBT@Pt Pdots in the presence of representative biologically relevant ions, and ROS in aqueous solutions. The concentration for each metal ion was $500 \mu \mathrm{M}$, and the $\mathrm{H}_{2} \mathrm{O}_{2}$ was $0.1 \mathrm{wt} \%$. 

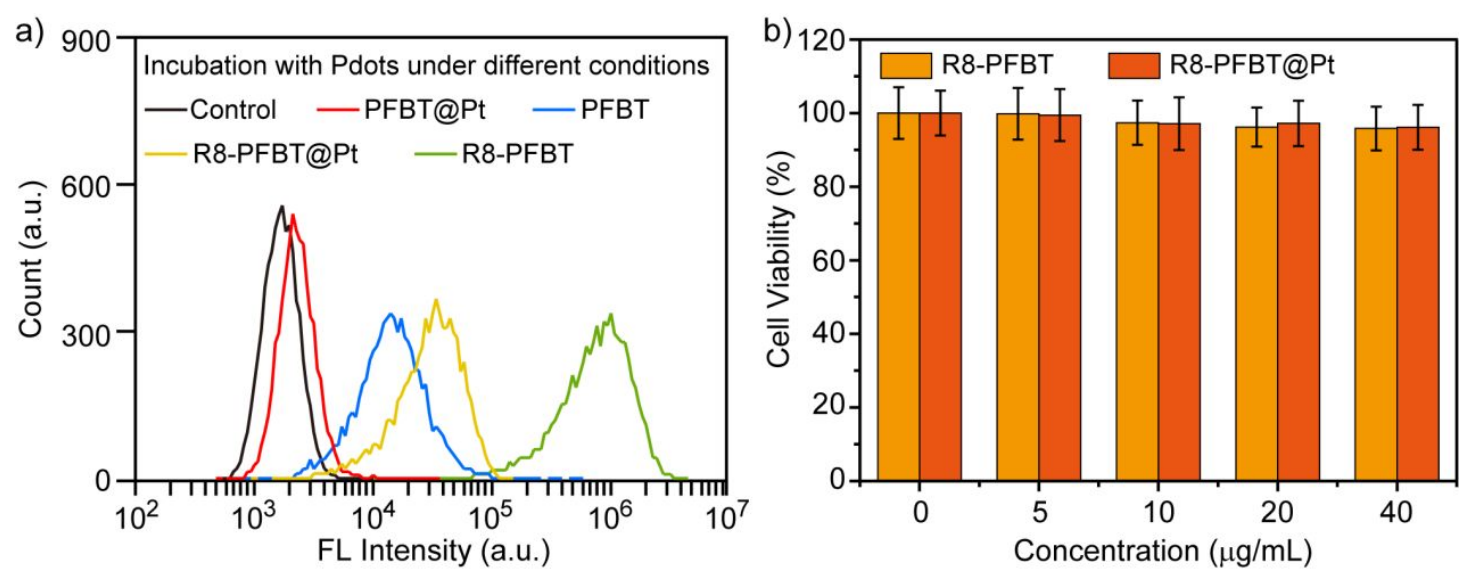

Figure S3. a) Brightness comparisons of MCF-7 cells labeled with PFBT@Pt Pdots, PFBT Pdots, R8-PFBT@Pt Pdots and R8-PFBT Pdots for 4 h by flow cytometry. b) Cytotoxicity assay of R8-PFBT Pdots and R8-PFBT@Pt Pdots.

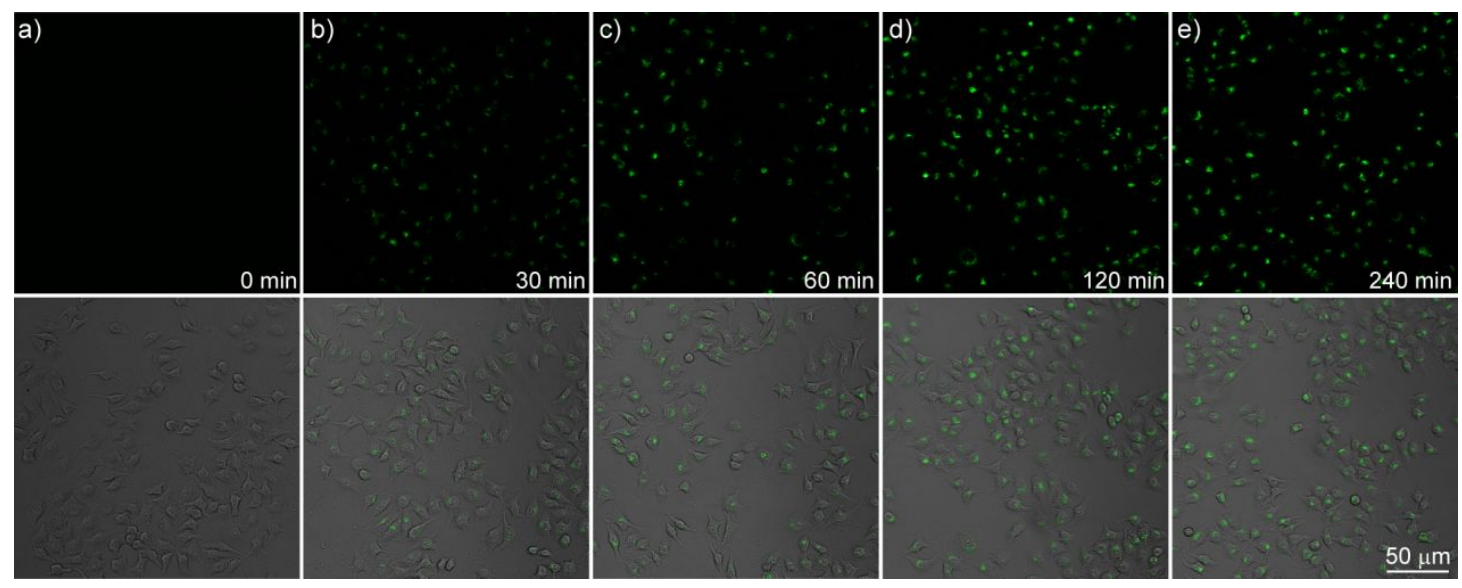

Figure S4. Typical confocal microscopy images with integrated intensity over Z-stacking images of MCF-7 cells labeled with R8-PFBT Pdots for a) o min, b) $30 \mathrm{~min}, \mathrm{c}) 60 \mathrm{~min}, \mathrm{~d}) 120 \mathrm{~min}$ and e) $240 \mathrm{~min}$. The scale bars represent $50 \mu \mathrm{m}$. 

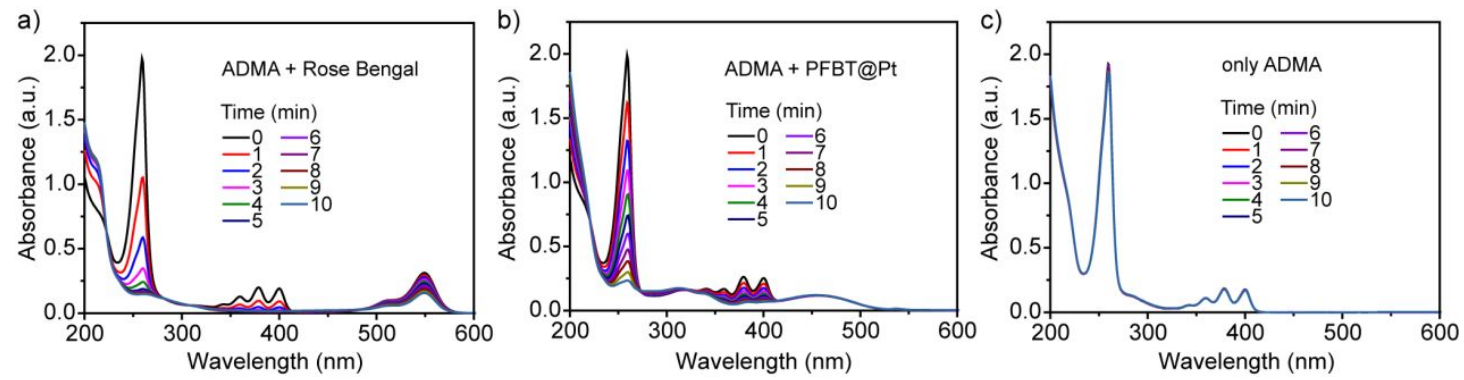

Figure S5. Changes of absorption spectra of a) ADMA in the presence of Rose Bengal, b) PFBT@Pt Pdots, and c) ADMA by light irradiation.

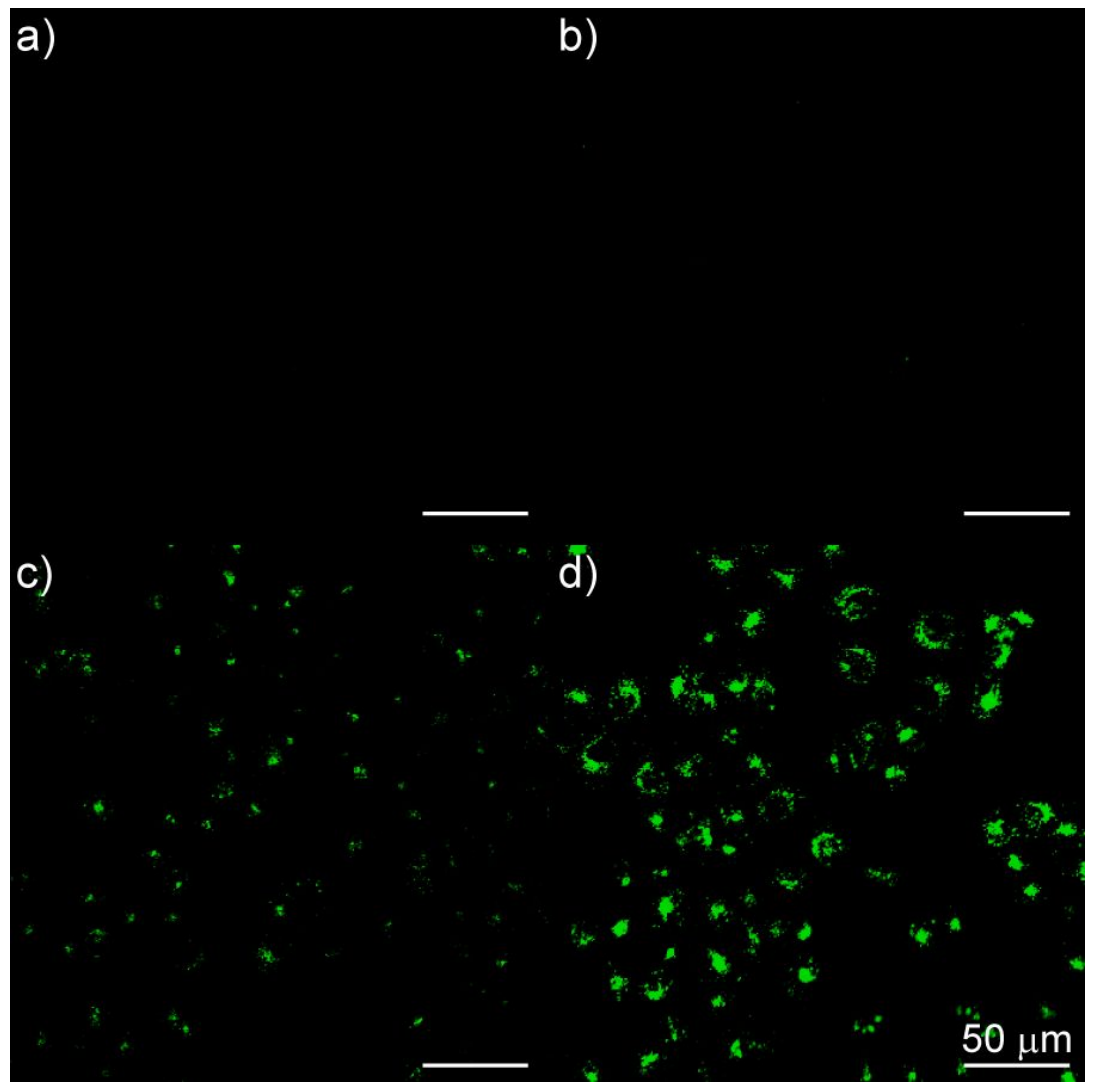

Figure S6. Confocal fluorescence imaging of MCF-7 cells treated with a) only DCFH-DA, b) DCFH-DA and the irradiation with $\sim 460 \mathrm{~nm}$ LED array for 10 min, c) DCFH-DA and the incubation with R8-PFBT@Pt Pdots and d) DCFH-DA, the incubation with R8-PFBT@Pt Pdots, and the irradiation with $\sim 460 \mathrm{~nm}$ LED array for $10 \mathrm{~min}$. The scale bars represent $50 \mu \mathrm{m}$. 